was performed and standardised utilising the validated 'BLUE protocol', performed by the same operator. Domains included demographics, respiratory comorbidities, diagnoses, and management plans prior to, and following TUS.

Results Of fifty patients scanned, TUS altered overall management in 22 (44\%). Primary diagnosis was changed in $26 \%$, treatments in $34 \%$, investigations in $28 \%$, and all three aspects in $18 \%$. TUS performed well in fluid balance decisions and identifying lung consolidation.

Patient groups where TUS would not alter management were identified, with reduced odds seen with pre-existing airways disease (odds ratio (OR) $0.37,95 \%$ confidence interval (CI) 0.12-1.17), and in patients with airways disease and wheeze on auscultation (OR 0.08, 95\%, CI 0.01-0.77).

Chest $x$-ray (CXR) reports differed from TUS findings in 12 (24\%). 21 (42\%) patients later underwent computed tomography (CT) examination with CT reports corresponding with positive TUS findings in $100 \%$, with no further emendation of diagnoses (excluding incidental findings). Data was not collected to assess the time implications of performing ultrasound on the consultation, and we acknowledge that not all Respiratory physicians are ultrasound trained thus limiting the provision of thoracic ultrasound.

Discussion The use of TUS impacted significantly on decisionmaking on the Respiratory PTWR. Unnecessary radiology requests, ionising radiation, and cost were avoided. Within the constraints of the study group, TUS seems less useful when assessing patients with pre-existing airways disease. TUS has excellent correlation with CT findings, outperforms CXR, appearing to offer a comprehensive, streamlined respiratory assessment at the 'front-door'.

With ultrasound becoming more accessible to clinicians, and with increasing demands on CT departments, we welcome discussion regarding regular use of TUS on the PTWR. Further data would be desirable to assess whether its use early in admission is correlated with a reduced length of stay and improved patient outcomes.

\section{P159 OUTCOME FROM INVASIVE VENTILATION FOR PATIENTS WITH LEARNING DISABILITY}

D McCourt, HM Tedd, TB Fretwell, T Doris, PB Messer. Newcastle Hospitals, Newcastle Upon Tyne, UK

\subsection{6/thorax-2021-BTSabstracts.268}

The annual learning disability mortality review (LeDeR) ${ }^{1}$ highlights that patients with learning disability (LD) are twice as likely to die of avoidable causes, with inappropriate ceilings of treatment being instigated in patients with LD: 23\% of 'Do Not Attempt Cardiopulmonary Resuscitation' forms documented inappropriate medical conditions such as a learning disability as the sole reason to not attempt resuscitation $^{1}$. The COVID19 pandemic has further highlighted this issue, with people with LD being at 6 times the risk of death from COVID19 than those without LD; people with LD aged 18-34 were 30 times more likely to die of COVID 19. ${ }^{2}$ However, there is little data out there to help support decision making around invasive ventilation in people with LD.

We aimed to explore the outcomes of patients with LD admitted to a single critical care unit for invasive ventilation
Methods We reviewed the notes of patients with learning disability requiring intubation and ventilation over a 5-year period (2016-2020). Data was retrospectively collected on survival, rates of tracheostomy insertion and requirement for long term ventilation (LTV) or cough augmentation.

Results 15 patients were identified with LD who required invasive ventilation. 93\% survived critical care admission. 7\% required tracheostomy with subsequent decannulation, whilst a further 7\% required long term tracheostomy. 40\% were commenced on LTV. 46\% required long term cough augmentation via cough assist device. 46\% have subsequently died, with a mean survival following critical care admission of 2.5 years. Mean duration from invasive ventilation in the survivor group is 5.5 years.

Conclusion We have demonstrated good outcomes in patients with LD admitted to critical care for invasive ventilation. Although there was a high requirement for LTV and cough augmentation following admission to critical care for invasive ventilation, we have demonstrated both good survival to discharge from critical care and good long term survival in this group. This small single centre study highlights the need for further research to aid decision making around escalation decisions in patients with LD.

\section{REFERENCES}

1. The learning disability mortality review (LeDeR): Annual report. 2020.

2. COVID19 deaths of people identified as having learning disabilities: summary. Public Health England. 2020.

\section{P160 COMMUNITY RESPIRATORY STAFF IN-REACH INTO CARE HOMES FINDS UNMET NEED AND ALLOWS OPTIMIZATION OF PATIENT CARE PLUS CARE HOME STAFF EDUCATION}

HS Hill, K Keeling, I Young, J Chandler, MCP Apps. North East London Foundation NHS Trust, Billericay, Essex, UK

\subsection{6/thorax-2021-BTSabstracts.269}

Introduction During the Covid epidemic the care of patients in care homes to optimize their therapy and prevent admission has become a national Priority. In the Basildon and Brentwood CCG area staff normally working in the community respiratory service were redeployed to visit all the care homes in the area and review all patients with respiratory disease to optimize therapy, advise flu immunisation and support their care.

Methods All care homes in the area of Basildon, Brentwood, Billericay and Wickford were visited and a review carried out on all patients resident there with respiratory disease. This included reviewing both notes and patients to find patients with respiratory disease. Therapy was reviewed and optimised, including obtaining new inhalers, spacer devices and rescue packs.

Results 163 patients were reviewed, of whom 116 had evidence of respiratory disease. 31 of these were already known to the community respiratory services and 85 were not known. $75 \%$ of patients needed a new salbutamol inhaler or aero-chamber spacer device. 58\% of patients did not have a rescue pack of antibiotics and steroids and this was provided. $85 \%$ of those not already known to the service required salbutamol,. A spacer and a rescue pack.

These were all provided. 